\title{
Muon-Pair Production by Atmospheric Muons in CosmoALEPH
}

\author{
F. Maciuc,,${ }^{1,+\dagger}$ C. Grupen, ${ }^{2}$ N.-O. Hashim,${ }^{2}$ S. Luitz, ${ }^{3}$ A. Mailov, ${ }^{2}$ A.-S. Müller, ${ }^{4}$ A. Putzer, ${ }^{5}$ H.-G. Sander, ${ }^{6}$ S. Schmeling, ${ }^{7}$ \\ M. Schmelling, ${ }^{1}$ R. Tcaciuc, ${ }^{2}$ H. Wachsmuth, ${ }^{7}$ Th. Ziegler, ${ }^{8}$ and K. Zuber ${ }^{9}$ \\ ${ }^{1}$ Max-Planck-Institut für Kernphysik, Saupfercheckweg 1, D-69117 Heidelberg, Germany \\ ${ }^{2}$ Fachbereich Physik, Universität Siegen, D-57068 Siegen, Germany \\ ${ }^{3}$ SLAC M/S 41, 2575 Sand Hill Road, Menlo Park, California 94025, USA \\ ${ }^{4}$ FZ Karlsruhe, Institut für Synchrotronstrahlung, D-76021 Karlsruhe, Germany \\ ${ }^{5}$ Kirchhoff-Institut für Physik, Universität Heidelberg, D-69120 Heidelberg, Germany \\ ${ }^{6}$ Fachbereich Physik, Universität Mainz, D-55099 Mainz, Germany \\ ${ }^{7}$ CERN, CH-1211 Geneva 23, Switzerland \\ ${ }^{8}$ Department of Physics, Princeton University, Princeton, New Jersey 08544, USA \\ ${ }^{9}$ Department of Physics, University of Oxford, Denys Wilkinson Building, Keble Road, Oxford OX1 3RH, United Kingdom
}

(Received 1 August 2005; published 19 January 2006)

\begin{abstract}
Data from a dedicated cosmic ray run of the ALEPH detector were used in a study of muon trident production, i.e., muon pairs produced by muons. Here the overburden and the calorimeters are the target materials while the ALEPH time projection chamber provides the momentum measurements. A theoretical estimate of the muon trident cross section is obtained by developing a Monte Carlo simulation for muon propagation in the overburden and the detector. Two muon trident candidates were found to match the expected theoretical pattern. The observed production rate implies that the nuclear form factor cannot be neglected for muon tridents.
\end{abstract}

DOI: 10.1103/PhysRevLett.96.021801

The trident interaction process occurs when a lepton $l_{1}$ produces a pair of leptons $\left(l_{2}^{+}, l_{2}^{-}\right)$in the field of nuclear charge Ze, thus $l_{1}\left(Z, l_{2}^{+} l_{2}^{-} Z\right) l_{1}$. Good theoretical estimates of the trident production rate date back to 1937 [1,2]. The first papers on this subject considered: small scattering angles, no exchange between diagrams [1], and only part of the leading order Feynman diagrams. The channels $e^{+} e^{-}$and $\mu^{+} \mu^{-}$were at this level indistinguishable except for the mass difference. About 30 years later the full QED formula of the differential trident cross section was computed [3-5]. However, there were two main difficulties to be solved: inclusion of the atomic and nuclear form factors and the simultaneous evaluation of the phase-space integral. Because of the high complexity of the full integral formula, the obtained QED result is impractical for most cross-section estimations. In practical applications, the result of a numerical integration of the QED matrix element over a multidimensional phase space is approximated by an analytical parametrization which is convenient for Monte Carlo (MC) simulations of leptonic tridents.

The early studies were motivated: by the necessity to compute radiative energy losses for charged particles (e.g., muon energy loss by emission of $e^{+} e^{-}$in the field of a nucleus), by the unknown nature of the muon [fermion or boson, see [6], concluded in [7] ], and by possible anomalous muon-muon interactions for $\mu\left(Z, \mu^{+} \mu^{-} Z\right) \mu$ [8].

Interference between amplitudes of different Feynman diagrams was estimated to be negligible for muons with energies higher than $10 \mathrm{GeV}$ [9]. Because the study presented in this Letter concerns high energy muons and due to the decreasing probability of muon tridents at low energy, interference terms were ignored.
PACS numbers: 13.40.Gp, 14.60.Ef, 25.30.Mr, 95.85.Ry

In 1967 Kelner [10] computed the QED differential cross section for tridents neglecting the nuclear form factor. The expression accounts for both $\mu\left(Z, \mu^{+} \mu^{-} Z\right) \mu$ and $\mu\left(Z, e^{+} e^{-} Z\right) \mu$ and its subsequent parametrization is the Bugaev-Kotov-Rozental formula [11] [BKR in [12]] that reduces the multidimensional integral to an analytical result. The formula has become a standard reference for professional muon transport Monte Carlo programs [e.g., [13] ] and for standard muon energy loss tables [14]. The BKR formula was later corrected by Kokoulin, Kelner and Petrukhin (KKP) [12] by the inclusion of a form factor for the nucleus. The QED result, with nuclear form factor, was parametrized in the KKP analytical formula. This final form considers implicitly the nucleus as a quasi-Coulomb scatterer with a Fermi function as form factor. The later result came correlated with the measurements of LVD and MACRO groups [15].

The BKR approximate cross-section formula gives good estimates for energy losses of muons in the radiative domain [14], where electron-positron production dominates. However, the formula leads to an overestimate in the observed event rate for the $\mu^{+} \mu^{-}$case [16]. This is due to the fact that the production of muon pairs has on average larger momentum transfers compared to $e^{+} e^{-}$production, and thus the nuclear form factor is more important in $\mu^{+} \mu^{-}$pair production. The corrected form, KKP, agrees with the data of [16]. It is the object of this Letter to show that the corrected formula has relevance for the results of the CosmoALEPH dedicated cosmic ray runs.

For tridents, the experimental signature is a primary track for the incoming muon and three quasicollinear tracks for the outgoing muons. In the laboratory reference 
system, the highly relativistic incident muons account for the forward production of the outgoing particles.

In the leading order Feynman diagrams of Fig. 1, the interaction "dynamics" is displayed. Subfigures II and III are radiative diagrams; I and IV are Bethe-Heitler diagrams. A phenomenological conclusion from the Feynman diagram analysis is the small deflection of the scattered muon coupled with low momentum transfer to the nucleus [6]. The muon momentum components transverse to the incident muon direction have values on the order of the muon mass [e.g., $300 \mathrm{MeV} / c$ on average for radiative diagrams [17] ]; thus, the angular distributions of the outgoing particles are strongly peaked forward, in the direction of the incident muon [3].

The results for photoproduction of heavy leptons, given by Tsai [18], can be applied in this analysis. The virtual photon emitted by the incoming muon (Fig. 1, diagram I) can be replaced by a real photon as in the lepton photoproduction. In this latter case, the muons have angular distributions with widths smaller but close to $m_{\mu} /(p\langle v\rangle)$, where $m_{\mu}$ is the muon mass, $p$ is the momentum of the incident muon, and $\langle v\rangle$ is the mean energy fraction transferred to the secondaries. For a typical trident, if the incident muon has an energy of $70 \mathrm{GeV}$, the mean energy fraction transferred to the pair is close to 0.2 and the angular distribution width has an average value of $0.4^{\circ}$. All these facts lead to very small scattering angles with respect to the incident muon direction. At production the trident muons have quasicollinear trajectories and these diverge as result of the $1.5 \mathrm{~T}$ strong magnetic field (Fig. 2). Evidence of this pattern was probed using the data of the CosmoALEPH experiment (observation of atmospheric muons with ALEPH, one of the main detectors at LEP).

From April 21 to 29, 1999, during a period without beam activity, the ALEPH detector [19] at CERN was used in
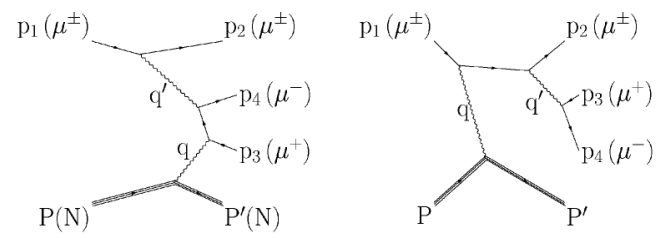

I. 1st Bethe-Heitler diagram

II. 1st radiative diagram
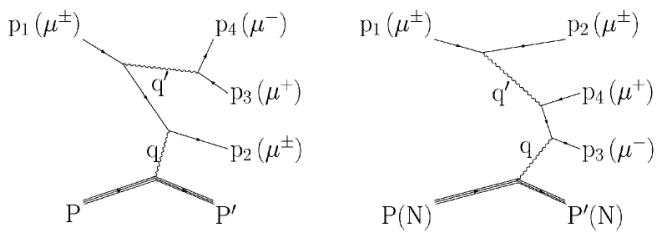

III. 2nd radiative diagram

IV. 2nd Bethe-Heitler diagram

FIG. 1. Muon trident leading order diagrams. dedicated cosmic ray runs as a muon spectrometer at a depth of 320 meters-water-equivalent (mwe) underground. The ALEPH subdetectors relevant for these measurements are the muon chambers, the hadron calorimeter (HCAL), the electromagnetic calorimeter (ECAL), and the time projection chamber (TPC). A single HCAL barrel module has 23 active sublayers. The CosmoALEPH trigger required a signal in at least 8 sublayers in one HCAL barrel module together with at least 8 confirmed hits in any of the three neighbor modules opposite to the first [20]. The muon detection efficiency per HCAL sublayer is $75 \%$ and the TPC reconstructs muon tracks with a $160 \mu \mathrm{m}$ spatial resolution in the $r \phi$ projection plane that is perpendicular to magnetic field in the cylindrically symmetric ALEPH. The calorimeters are sufficiently massive to stop any charged or neutral-hadronic secondaries produced by muons. They do let the high energy muons pass; thus a hadron undergoing decay to muon generates a muon track in the calorimeters.

In approximately $1 \times 10^{6}$ events with at least one muon, as tagged by ALEPH, only close to 5000 events contain more than one observed muon. Among these, there are events in which atmospheric muon bundles are detected. These contain almost parallel tracks, because the vertex of the parent hadron is high in the atmosphere, in the kilometer range [21,22], and the muon and hadron directions are almost the same at the energies involved. The cutoff for 320 mwe overburden is close to $75 \mathrm{GeV}$. Besides these atmospheric muon bundle events, there are events where a muon undergoes an interaction in the detector and secondary particles are produced. Electrons, positrons, and photons are produced in knock-on, bremsstrahlung, and pair production processes in the calorimeters, and could be observed (only the charged component) in the TPC as remnants of electromagnetic showers, or as direct products from reactions in the innermost part of ECAL. Since the ECAL has a thickness of 21.5 radiation lengths, the only

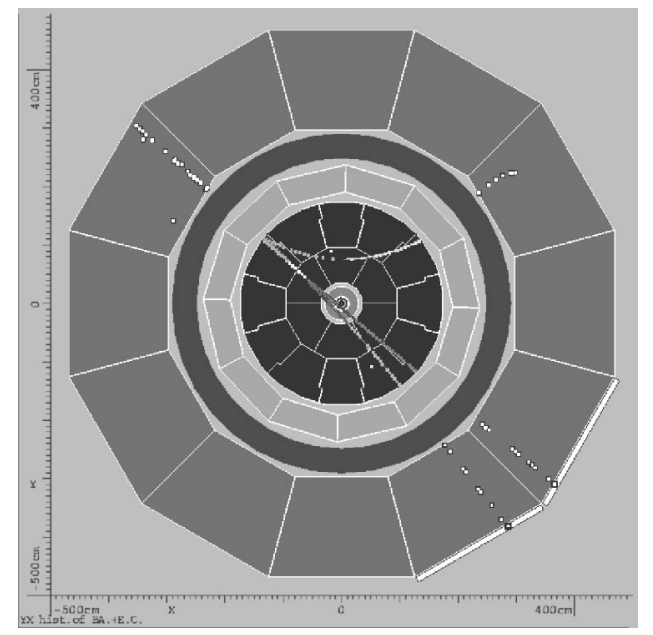

FIG. 2. Trident candidate in ALEPH. 
reactions that lead to clear tracks in HCAL besides the incident muon tracks are the photonuclear and $\mu^{+} \mu^{-}$pair production. The electron-positron pair production has a track pattern in the TPC similar to the $\mu^{+} \mu^{-}$pair production. However, the absence of HCAL tracks that would extend the electron and positron TPC tracks gives a clear distinction between electron tridents and muon-only tridents.

The problem of separating a trident $\mu\left(Z, \mu^{+} \mu^{-} Z\right) \mu$ from photonuclear interactions is due to the fact that the produced pions have a mass close to muon mass and they can decay to muons. Photonuclear interactions are more probable than tridents, and it is possible that in some photonuclear interactions two pions of opposite charge are produced, which subsequently decay into two muons, leading to an event similar to a trident. However, if peculiar aspects of each event are considered, such as large secondary muon energy, the photonuclear hypothesis becomes much less probable than the trident hypothesis. To have a clear track in the bottom calorimeter modules, a high energy pion has to penetrate through part of HCAL with $1.2 \mathrm{~m}$ of iron thickness corresponding to 7.2 interaction lengths and all ECAL with $11 \mathrm{~cm}$ of lead or one interaction length thickness. At the given energies the pion-decay probabilities over the distance traveled through the detector are small compared to the interaction probability. Therefore, considering only the latter, the probability that two final state pions from a 3-prong inelastic event are mistaken with muons is around $10^{-7}$. With only three $3-$ prong events found in the data sample, it follows that hadronic background to tridents candidates is negligible.

Following the above criteria, the 5000 multimuon events were reduced to only two trident candidates. Both events contain very high energy muon tracks. The probability that the events are results of photonuclear interactions is negligible. There is no evidence of hadronic interaction and the energies are high enough to reject the pion-decay hypothesis. The muon chambers are triggered by the exiting particles. The analysis was done to select trident candidates with a vertex in the detector or in the overburden layers.

To simulate the muon propagation in detector and overburden, a Monte Carlo calculation was developed. The input is provided by the muon direction, energy, and position as measured by the TPC. All reconstructed muon tracks are used.

The MC calculation extrapolates the energy of each recorded muon from the detector level back to the surface. The tridents are generated along the way and the resulting muons propagate, if sufficiently energetic, down to ALEPH's TPC, where their energy is measured. The systematic uncertainties of the simulation were estimated by varying the granularity of the material description and the numerical treatment of the underlying dynamics and found to be significantly smaller than the final errors. Given the selection criteria of candidates, the MC overall sample is divided into two subsamples. The first subsample contains tridents where one muon is stopped in the detector. The second subsample contains tridents for which all muons exit the detector, thus crossing ALEPH from one side to the other if the trident vertex is in the overburden, and from the vertex to the opposite side if the tridents are produced in the detector. One candidate was found in each of the two categories. Other MC tridents with less clear signatures are neglected by this analysis.

In order to get a statistical description of the characteristics of muon trident production in CosmoALEPH, the simulation of the entire muon sample through overburden and detector was repeated 2000 times. The results are shown in Figs. 3 and 4 for tridents with vertex in the detector. In the overburden, the expected number of tridents is close to 2, but as these MC tridents have a low energy secondary, the trident pattern is expected to be obliterated by the presence of the strong magnetic field $(1.5 \mathrm{~T})$. This makes the distinction between these muon tridents and other events (e.g., muon bundles from the same shower) virtually impossible. For the tridents with vertex in the detector layers the pattern is clear. In Fig. 3, the expected number for the latter case is determined by a Poisson-like distribution with 2.6 mean value. This value is consistent with the two candidates found by CosmoALEPH. The BKR estimate should be at least 5 times higher [12], and appears to be overruled by the data.

The first trident candidate shown in Fig. 2 has one muon that stops in a HCAL sublayer. The vertex is located in an upper module of HCAL, and the muon tracks in the TPC are good quality distinct tracks. The energy of the incident particle (neglecting the recoil of the iron nucleus) is approximately $57 \mathrm{GeV}$. As shown in Fig. 4, the energies of the outgoing muons are consistent with the Monte Carlo ex-

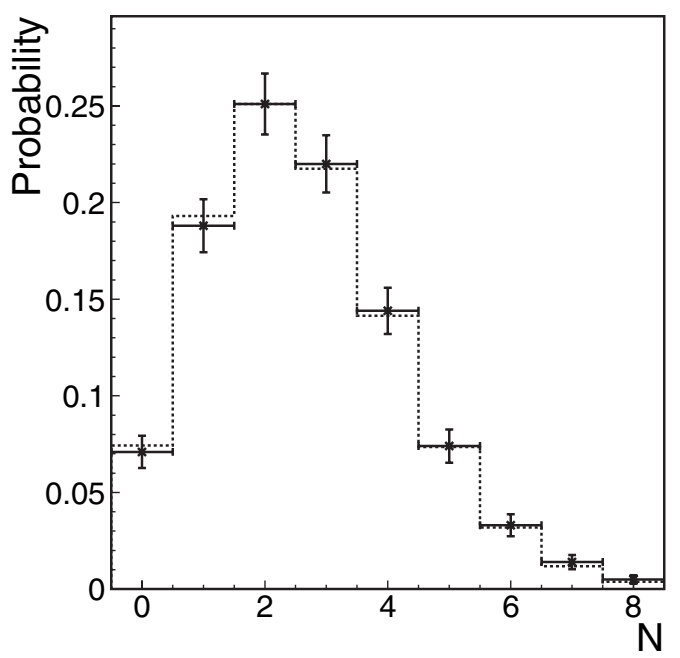

FIG. 3. MC probability distribution for the total number of tridents with vertex in the detector (crosses), Poisson distribution with 2.6 average (histogram). 


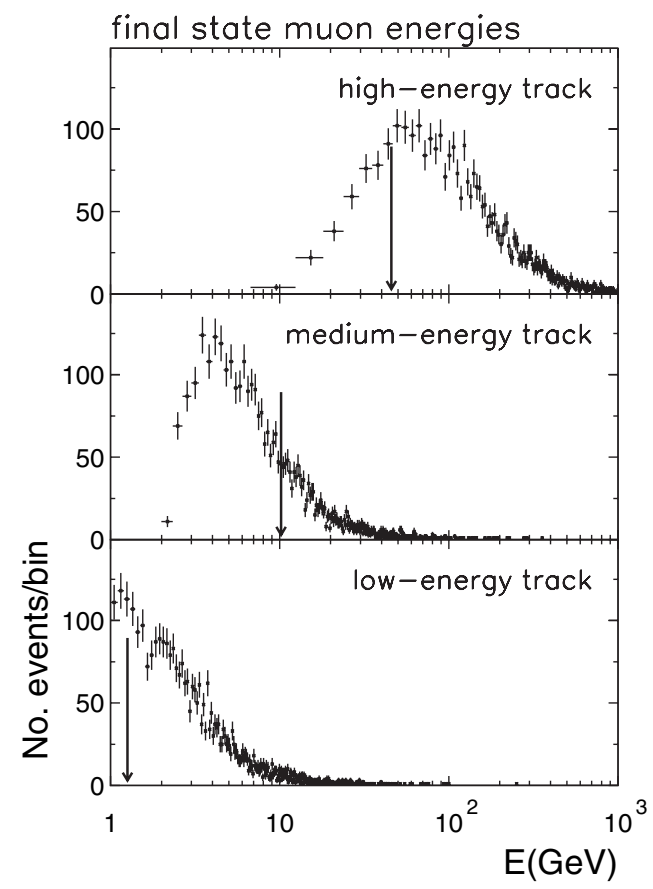

FIG. 4. MC distribution for the trident muon energies in the TPC: the arrows mark the position of the corresponding candidate energies (first candidate); the error bars correspond to bin width and statistic errors.

pectations for typical trident events in CosmoALEPH. All three final state muons have energies close to the most probable values. The measured energies of $45.8 \pm 4.4$, $10.2 \pm 0.33$, and $1.26 \pm 0.02 \mathrm{GeV}$ are within $29 \%, 58 \%$, and $8.3 \%$ confidence level regions around the peaks of the Monte Carlo distributions.

The second event has all muons crossing the detector from the vertex to the opposite side of ALEPH. The energy of the assumed primary muon is not reconstructed due to the high energy and the partial overlap between the track of one secondary and the primary track. The same holds true for the higher energy secondary muon. Because of overlap of the two highest energy tracks, the only reliable value for the reconstructed energy is the energy of the low energy secondary, and this is determined to be $13 \pm 1 \mathrm{GeV}$.

Within the low statistics of two events, the CosmoALEPH data appear to be consistent, both in event number and particle energies, with the theoretical expectation from KKP $[12,16]$. The BKR estimate $[11,16]$, neglecting nuclear from factors, is strongly disfavored. An extended description of the CosmoALEPH events is given in the graduation thesis of one of the authors [23].

The authors gratefully acknowledge the help of the ALEPH Collaboration, in particular, the shift crew mem- bers of ALEPH, without whose support the data taking would not have been possible. This work has been funded by the Deutsche Forschungsgemeinschaft under Grant No. DFG/Gr/1796/1-3.

*On leave from Fachbereich Physik, Universität Siegen, D-57068 Siegen, Germany.

${ }^{\dagger}$ Email address: Florin.Maciuc@mpi-hd.mpg.de

[1] G. Racah, Nuovo Cimento 14, 93 (1937).

[2] J. A. Wheeler and W.E. Lamb, Jr., Phys. Rev. 55, 858 (1939).

[3] S. J. Brodsky and S.C.C. Ting, Phys. Rev. 145, 1018 (1966).

[4] J.D. Bjorken and M.C. Chen, Phys. Rev. 154, 1335 (1967).

[5] G. R. Henry, Phys. Rev. 154, 1534 (1967).

[6] M. J. Tannenbaum, Phys. Rev. 167, 1308 (1968).

[7] J. J. Russell et al., Phys. Rev. Lett. 26, 46 (1971).

[8] M. J. Tannenbaum, NAL Summer Study Report No. B.268-32 1968 (unpublished).

[9] S. R. Kelner, Phys. At. Nucl. 61, 448 (1998).

[10] S. R. Kelner, Sov. J. Nucl. Phys. 5, 778 (1967).

[11] E. V. Bugaev, Y.D. Kotov, and I. L. Rozental, Cosmic Muons and Neutrinos (Atomizdat, Moscow, 1970) (in Russian).

[12] R. P. Kokoulin, S. R. Kelner, and A. A. Petrukhin, in Proceedings of the International Cosmic Ray Conference, Salt Lake City, 1999, edited by D. Kieda et al. (American Institute of Physics, Melville, NY, 2000), Vol. 2, p. 20.

[13] R. Brun et al., computer code GEANT3.15, CERN Report No. DD/EE/84, 1987 (unpublished).

[14] W. Lohmann, R. Kopp, and R. Voss, CERN Yellow Report No. EP/85-03 (unpublished).

[15] V. A. Kudryavtsev et al., Phys. Lett. B 471, 251 (1999).

[16] V. B. Anikeev et al., in Proceedings of the International Cosmic Ray Conference, Hamburg, 2001, edited by K.-H. Kampert et al. (Copernicus Gesellschaft, Lindau, 2001), Vol. 3, p. 962.

[17] V. Ganapathi and J. Smith, Phys. Rev. D 19, 801 (1979).

[18] Y. S. Tsai, Rev. Mod. Phys. 46, 815 (1974).

[19] D. Buskulic et al. (ALEPH Collaboration), Nucl. Instrum. Methods Phys. Res., Sect. A 360, 481 (1995).

[20] S. Schmeling, CosmoALEPH Handbook, CosmoALEPH 99-001, CERN print, 1999 (unpublished).

[21] C. Grupen, Astroteilchenphysik (Friedr. Vieweg \& Sohn, Braunschweig, Wiesbaden, 2000).

[22] C. Grupen, Astroparticle Physics (Springer, New York, 2005).

[23] F. Maciuc, Diploma thesis, University Siegen, 2004 (unpublished); http://www.hep.physik.uni-siegen.de/ grupen/ florin.ps.gz. 